\title{
Comportamiento informativo de personas adultas mayores
}

\author{
Patricia Hernández Salazar \\ Universidad Nacional Autónoma de México, Instituto de Investigaciones Bibliotecológicas y de \\ la Información, México, Cd. Mx., México \\ phs75599@unam.mx
}

\section{DOI: https://doi.org/10.26512/rici.v12.n2.2019.21781}

Recebido/Recibido/Received: 2018-12-15

Aceitado/Aceptado/Accepted: 2019-01-08

Resumen: El propósito de este trabajo es describir el proyecto: antecedentes, preguntas de investigación, objetivos, marco teórico, y los métodos, técnica e instrumentos. La investigación sigue el enfoque cognitivo y es de corte cualitativo, la intención es acercarse a las personas adultas mayores, para examinar sus actividades y emociones, desde y con ellos. El modelo teórico que se seguirá es el desarrollado por T.D. Wilson, Modelo de comportamiento informativo (2000), integra las fases por las que pasa un sujeto para resolver un problema de información: delimitación de necesidades de información, comportamiento en la búsqueda, y procesamiento y uso. Los métodos y técnica cualitativos son: observación directa, grupo focal y entrevista a profundidad. Se espera: entender el comportamiento de los adultos mayores ante la información; proponer servicios de información que resuelvan sus necesidades; e incidir en la generación de políticas públicas a favor de estas comunidades.

Palabras clave: Comportamiento informativo. adultos mayores. enfoque cognitivo. estudio de usuarios. tendencia cualitativa.

\section{Informative behavior of older adults}

Abstract: The purpose of this paper is to describe the project: background, research questions, objectives, theoretical framework, the methods, technique and instruments. The research follows the cognitive approach and it is mainly qualitative, the intention is to come close to the older adults, to examine their activities and emotions, from and within them. The theoretical model that will be followed is that developed by T.D. Wilson Information Behavior Model (2000), which integrates the phases each subject goes through to solve an information problem: delimitation of information needs, seeking behavior, and processing and use. The qualitative methods and technique are: direct observation, focus group and indepth interview. The expected outcomes are: to understand the information behavior of older adults; propose information services that meet their needs; and influence public policies in support these communities.

Key words: Information behavior. older adults. cognitive view point. qualitative approach. user studies.

\section{Comportamento informativo de idosos}

Resumo: O objetivo deste artigo é descrever o projeto: antecedentes, questões de pesquisa, objetivos, referencial teórico, e método, técnica e instrumentos de medida. A pesquisa é focada a partir de uma abordagem cognitiva de tipo qualitativa, a intenção é se aproximar aos adultos idosos para examinar suas atividades e emoções, a partir deles e com eles. O modelo teórico que será seguido é o desenvolvido por T.D. Wilson, Modelo de comportamento informativo (2000), que integra as fases pelas quais um sujeito passa para resolver um problema de informação: delimitação de necessidades de informação, comportamento na busca e processamento e uso. O métodos e técnica qualitativo são: observação direta, grupo focal, e entrevista em profundidade. Os resultados esperados são: entender o comportamento 
informacional dos idosos; propor serviços de informação; e influir nas políticas públicas para contribuir ao melhoramento da idosos.

Palavras-chave: Comportamento informacional. idosos. abordagem cognitiva. estudo de usuários. perspectiva qualitativa.

\section{Introducción}

A nivel internacional se ha realizado una gran cantidad de estudios de usuarios que abarcan diversas comunidades. Una que ha sido poco estudiada es la conformada por los adultos mayores. Comunidad que presenta características biológicas, psicológicas, económicas y sociales particulares, que la hacen proclive a subutilizar o no utilizar los recursos de información disponibles en las distintas instituciones que cubren sus necesidades. Para corregir esta carencia, se está realizando una investigación sobre el comportamiento informativo de adultos mayores, cuyo objetivo es identificar la totalidad de las actividades y los elementos que conforman su comportamiento en relación con la información dentro de sus espacios cotidianos. El propósito de este trabajo es describir el proyecto, para cubrirlo se presentan seis apartados: antecedentes, preguntas de investigación, objetivos, marco teórico, los métodos, técnicas e instrumentos y algunas conclusiones.

Un aspecto tácito para identificar a un adulto mayor está vinculado con su papel social, la percepción que la sociedad tiene de ellos. Generalmente se consideran como sujetos pasivos, dependientes y que requieren ser cuidados por otras personas, lo que les confiere un cierto grado de vulnerabilidad y exclusión. Estas condiciones pueden ser paliadas con el uso de información.

\section{Antecedentes}

Para ubicar la problemática que da origen a este proyecto, se consideraron cuatro vertientes: situación social de las personas adultas mayores, programas internacionales que los apoyan, la investigación que se ha realizado en bibliotecología y ciencia de la información y el escenario en México.

\subsection{Situación social}

De acuerdo con datos de la Organización Mundial de la Salud (OMS) la población con más de 60 años va en aumento, una proyección de 35 años (entre el 2015 y el 2050) indica que de 900 millones pasará a 2000 millones, se doblará el porcentaje del $11 \%$ al 22\% de la población mundial. (Organización Mundial de la Salud. [online] Disponible en https://www.who.int/features/factfiles/ageing/es/ ). Cabe aclarar que este aumento no ha sido 
consistente en todos los países, depende del contexto o entorno. Un ejemplo es la esperanza de vida, en los países desarrollados es mayor que en los denominados en desarrollo, a continuación, aparecen algunas cifras (Tabla 1).

Tabla 1 - La esperanza de vida

\begin{tabular}{|ll|}
\hline Países en desarrollo: & \\
Nigeria & 54 años \\
Pakistán & 68 años \\
México & 76 años \\
\hline
\end{tabular}

$\begin{array}{ll}\text { Países desarrollados: } & \\ \text { EUA } & 80 \text { años } \\ \text { Noruega } & 82 \text { años } \\ \text { Japón } & 85 \text { años }\end{array}$

Fuente: Index Mundi. Disponible en: https://www.indexmundi.com/map/?v=30

A partir de estos datos se puede apreciar que existen diferencias entre países en desarrollo y desarrollados y entre los miembros del mismo grupo, en el primero, entre Nigeria y México hay una diferencia de 22 años; en el segundo sólo es de 5 años. Estas cifras muestran que el desarrollo de la calidad de vida varía y por consiguiente la situación social.

Sin embargo, el aumento en la esperanza de vida no evita la disminución de las capacidades físicas y mentales propias del envejecimiento, por lo que los gobiernos de los países tienen que hacer inversiones mayores para su adecuada atención. Tanto el aumento en la población como en la esperanza de vida promueven que los adultos mayores vivan en condiciones de vulnerabilidad, exclusión y en algunas ocasiones maltrato y violencia por parte de la sociedad.

\subsection{Programas internacionales a favor de adultos mayores}

Una preocupación reiterada por varias instancias internacionales y regionales es atender las necesidades de estas comunidades, para que logren una mayor calidad de vida y aumenten su capacidad funcional, participación social y en general su bienestar. Existen varias iniciativas y organismos que se han ocupado de esto, todas integran estrategias sociales y económicas, por lo que surgió el interés de identificar si en su contenido existe alguna relacionada directamente con la información, a continuación, se describen de forma somera los programas más destacados generados por la Organización de las Naciones Unidas (ONU):

a. Plan de Acción Internacional de Viena sobre el Envejecimiento (1982). Los aspectos que motivan su creación son de corte demográfico, sociales y humanitarios. Enfatiza la colaboración de los gobiernos de todas las naciones para que haya resultados. Resaltan dos objetivos que es posible relacionar con la información y su uso: 
d) Presentar alternativas y opciones de política que sean compatibles con los valores y metas nacionales y con los principios reconocidos internacionalmente con respecto al envejecimiento de la población y a las necesidades de las propias personas de edad; y e) Alentar el desarrollo de una enseñanza, una capacitación y una investigación que respondan adecuadamente al envejecimiento de la población mundial y fomentar el intercambio internacional de aptitudes y conocimiento en esta esfera. (Plan de Acción..., 1982. [online] Disponible en: http://www.sld.cu/galerias/pdf/sitios/gericuba/plan de accion internacional de vie na sobre el envejecimiento.pdf)

Presenta siete esferas de preocupación dentro de las que enmarca 51 recomendaciones: salud y nutrición, protección de los consumidores ancianos, vivienda y medio ambiente, la familia, bienestar social, seguridad del ingreso y el empleo, y educación.

b. Principios de las Naciones Unidas en favor de las Personas de Edad (1991). Considera cinco aspectos: independencia, participación, cuidados, autorrealización y dignidad, se destacan los siguientes (ver Fig. 1).

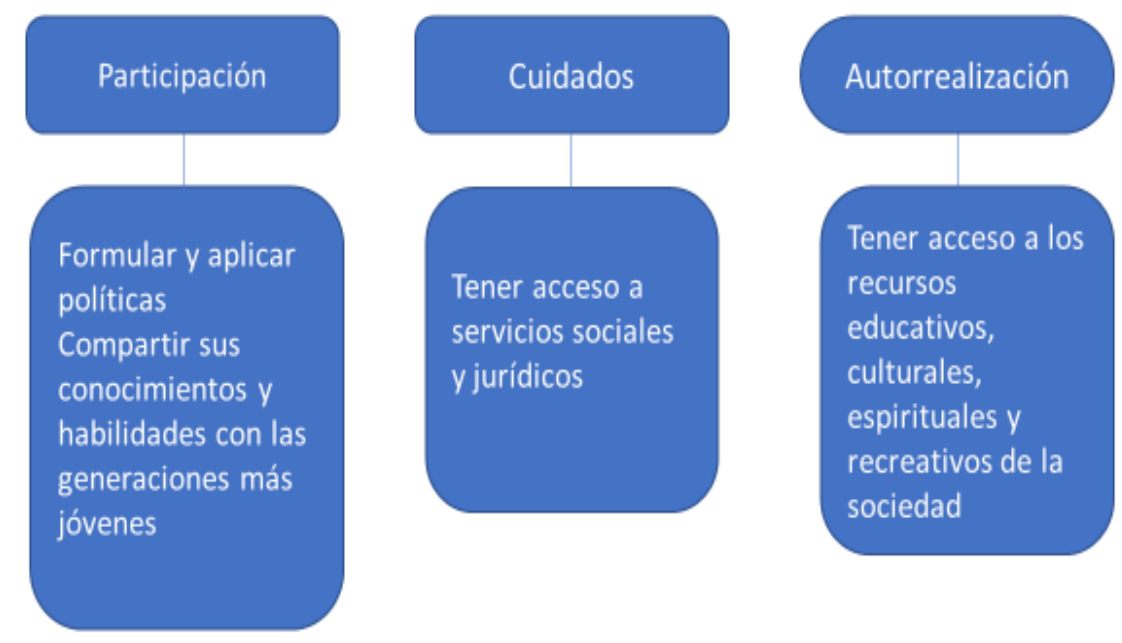

\section{Fig. 1. Aspectos relacionados con la información.}

c. Proclamación sobre el Envejecimiento (1992). Resultado de la 41á. Sesión plenaria, para la cual se convocó a una conferencia internacional sobre el envejecimiento, insta a retomar el Plan de Acción Internacional de Viena sobre el Envejecimiento, entre las estrategias aparece:

j) A que aliente a la prensa y a los medios de información pública a desempeñar una función central en la toma de conciencia sobre el envejecimiento de la población y las cuestiones conexas [...] [online] (Disponible en: https://undocs.org/es/A/RES/47/5, p. 13). 
En cuanto a la violencia existen declaraciones e instancias que se están ocupando del tema: Declaración de Toronto para la Prevención Global del Maltrato de las Personas Mayores (2002); Red Internacional de Prevención del Abuso y Maltrato en la Vejez (INPEA por sus siglas en inglés de International Network for the Prevention of Elder Abuse) y la Convención Interamericana sobre la Protección de los Derechos Humanos de las Personas Mayores, promovida por la Organización de Estados Americanos.

Se aprecian dos posturas con respecto a la información, una hacia la sociedad para que tenga una percepción diferente de los ancianos y otra en relación con identificar sus necesidades y cubrirlas. En lo que todos coinciden es en realizar investigaciones que apoyen su inclusión, disminuyan su vulnerabilidad, promuevan su independencia y autorrealización y que la sociedad los trate con dignidad.

\subsection{Investigación en bibliotecología y ciencia de la información.}

El estudio de estas comunidades empieza en la década de los setenta, el objetivo en esa época fue identificar sus necesidades de información (Hernández Salazar, 2014). En las dos últimas décadas se han hecho investigaciones relacionadas con las tecnologías de computación (TAK, HONG, 2005; SCHELLING, SEIFERT, 2010; SMITH, 2014) y las tecnologías de información y comunicación (TIC) (CHU, et al., 2009; IHM, HSIEH, 2015;). Principalmente se han tratado los siguientes temas: necesidades de información; comportamiento en la búsqueda; servicios; medios de comunicación masiva; habilidades informativas; aprendizaje para toda la vida y tecnologías de información y comunicación.

Los países que más lo han trabajado son: Canadá (ALLEN, WILKINSON, 1990; WICKS, 2004); Estados Unidos de Norteamérica (TAK, HONG, 2005; CHU et al., 2009; SMITH, 2014; IHM, HSIEH, 2015); Finlandia (NIEMELA, HUOTARI, KORTELAINEN, 2012); Reino Unido (DEE, BOWEN, 1986; BARRETT, 2000, 2005; GOODMAN, BREWSTER, GRAY, 2002; GODFREY, JOHNSON, 2009; BROWNSELL, BLACKBURN, HAWLEY, 2012), y Suiza (SHCELLING, SEIFERT, 2010).

Un elemento que resulta especialmente relevante para examinar a esta comunidad es el espacio en el que residen o al que asisten cotidianamente. El lugar de residencia se refiere al inmediato, en el que habitan, como puede ser una casa (ya sea que vivan solos o con otros familiares) o residencias para adultos mayores (también llamadas asilos, casas, o ancianatos). En cuanto a los sitios a los que asisten cotidianamente están clubes, casas de cultura, iglesias o bibliotecas, entre otros. Atendiendo a esta categoría los estudios se han situado en términos generales en bibliotecas públicas (DEE, BOWEN, 1986; ALLEN, WILKINSON, 1990; HEIM, WALLACE, 1990) e instituciones que ofrecen servicios comunitarios y residencias para ancianos (WICKS, 2004). 
El comportamiento informativo y el uso de la información son subcampos que se han dejado fuera, el primero entendido como la totalidad del comportamiento de los sujetos en relación con la información, y el segundo como la transformación que se realiza en el acervo de conocimientos de una persona, al analizar, sintetizar, asimilar, aprehender y acomodar nueva información en su cadena de conocimientos.

\subsection{Escenario en México.}

En este país no se han hecho investigaciones sobre el comportamiento informativo de dichas comunidades, a pesar de que ha habido un incremento numérico de esta población y se enfrentan a problemas de exclusión y vulnerabilidad.

La situación demográfica y social en este país se corresponde con la internacional, ha habido un aumento, entre las décadas de 1970 y 1990 el porcentaje de adultos mayores pasó de 5.6\% a 6.2\%; para 2017 dicho porcentaje fue de 10.5\%. Según el censo de 2010 existían 10,055,379 personas de 60 años y más, para 2017 la cifra aumentó a 12,973,411. (Contacto hoy Edición Mundial. [online] Disponible en: https://contactohoy.com.mx/mas-de-12-millones-depersonas-en-mexico-son-adultos-mayores-inegi/) .

Como se apuntó en párrafos anteriores, el contexto es determinante para establecer su situación, cada país tiene su propia legislación y programas. En México se cuenta con dos leyes, la Ley de los Derechos de las Personas Adultas Mayores publicada por primera vez en 2002, la cual sigue los principios propuestos por la ONU, agrega equidad y trato preferente. En relación con la información, en su Artículo 6, inciso II se lee:

II. Información: Las instituciones públicas y privadas, a cargo de programas sociales deberán proporcionarles información y asesoría tanto sobre las garantías consagradas en esta Ley como sobre los derechos establecidos en otras disposiciones a favor de las personas adultas mayores [...] (Ley de los Derechos de las Personas Adultas Mayores, 2016, $\quad$ p. $\quad 6.6$ [pdf] https://www.gob.mx/cms/uploads/attachment/file/175189/245 221116.pdf ).

Y la Ley de albergues privados para personas adultas mayores del Distrito Federal (actualmente Ciudad de México) creada en 2009, la que los clasifica estos espacios como albergues y centros de día, públicos y de asistencia social y privados, lucrativos y no lucrativos, integra lo siguiente:

Artículo 5:

II. Vigilar que los administradores de albergues proporcionen información sobre la cobertura y características de los servicios que prestan para los adultos mayores [...] (Ley de albergues privados para personas adultas mayores del Distrito Federal, 2009, p. 3. [pdf] Disponible en: http://aldf.gob.mx/archivoc3c84deb4108970eaee0851d7bed9198.pdf) 
Con respecto a los programas nacionales y estatales dirigidos a esta población, existen varios, que pueden clasificarse en tres rubros:

- Apoyo económico: Pensión para Adultos Mayores; Pensión alimentaria para Personas Adultas Mayores de 68 años; y Créditos para personas mayores.

- Instituciones y espacios: Centros de atención integral con énfasis en servicios de salud; Centros de Capacitación para el Trabajo y Ocupación del Tiempo Libre; Albergues y estancias diurnas; y Clubes comunitarios.

- Servicios. Su objetivo es mantener un vínculo con la población adulta mayor: Actividad física y deporte; Servicio de Turismo Social; Visitas Médicas Domiciliarias; Visitas Domiciliarias; y Sistema Alerta Social. (MEXICO. Secretaría de Desarrollo Social. Guía de programas sociales. [online] Disponible en: https://www.gob.mx/cms/uploads/attachment/file/119689/GPS 2016.pdf .)

Sobresale el hecho que no existe algún programa que puntualmente exprese el ofrecimiento de servicios o recursos de información, cuando de suyo estos resolverían o apoyarían en la solución de problemas de cualquier índole, lo que abonaría al objetivo de mejorar sus condiciones de vida y bienestar.

\section{Preguntas de investigación}

Concretamente, la problemática apunta a dos causas: condiciones sociales que los identifican como una población vulnerable y poco reconocida y carencia de estudios que permitan identificar sus características vinculadas con el uso de información, que apoye su cabal integración como sujetos activos e independientes, mediante el reconocimiento de la importancia de la información para resolver sus actividades de la vida diaria.

Como se anotó un elemento esencial para estudiarlos es el espacio que habitan o al que acuden cotidianamente, por lo que los problemas de investigación se circunscriben al aspecto espacial. Las interrogantes planteadas son:

- ¿A qué tipo de problemas se enfrentan en su vida y espacios cotidianos las personas adultas mayores?

- ¿Qué actividades realizan y a qué instituciones se acogen para resolver dicha situación problemática?

- ¿Cómo transforman cognitivamente la información?

\section{Objetivos}

General. Identificar la totalidad de los elementos que conforman el comportamiento de comunidades de adultos mayores en relación con la información dentro de sus espacios cotidianos.

\section{Particulares:}


1. Establecer su perfil de necesidades de información.

2. Identificar su comportamiento en la búsqueda y recuperación de información.

3. Precisar los procesos cognitivos que realiza para asimilar y aplicar la información a la solución de sus problemas cotidianos.

\section{Marco teórico}

La literatura sobre el tema apunta un abordaje ecléctico, incluso en algunos textos no se definen los aspectos teóricos. El desarrollo de esta investigación está basado en el enfoque cognitivo que surgió a finales de la década de los setenta y se consolidó en los ochenta y noventa del siglo pasado, principalmente con autores como N. Belkin $(1978,1982,1990)$, B. C. Brookes $(1974,1975,1977,1980,1981)$, T. D. Wilson $(1981,1997,1999$ y 2000) y R. Todd $(1996,1999 a$, 1999b). Este enfoque toma como principio fundamental al sujeto, concretamente sus procesos cognitivos, considera que una persona atiende a sus imágenes del mundo para definir sus necesidades de información, buscarla, recuperarla y utilizarla. El ser humano se percibe en constante construcción, sus requerimientos se circunscriben a ciertas situaciones que pueden cambiar. Para estudiar al usuario mediante este enfoque es necesario valerse del lenguaje y lo discursivo como una forma de entenderlo.

Se retoman tres vertientes teóricas: el concepto de adulto mayor, el modelo de estudio y el uso de información.

\subsection{Concepto de adulto mayor.}

En términos generales se define desde dos perspectivas, una biológica y otra económica. La primera atiende a los cambios físicos y mentales que se presentan con el paso del tiempo, un adulto mayor es aquel que ha perdido o le han disminuido ciertas capacidades físicas, anatómicas o fisiológicas (visión, audición, motricidad), lo que altera también su estado psicológico. La relación entre la edad cronológica y biológica es lo más difícil de establecer, puesto que depende de las condiciones económicas, educativas, tecnológicas, científicas, sociales, culturales, alimenticias, ambientales y de servicios de salud que prevalezcan en cada país o región geográfica, es decir su contexto. Se podría inferir que cada país tendría su propio límite cronológico para denominar a las personas adultas mayores, sin embargo, existe una convención aceptada en diversas regiones que la edad para ser una persona adulta mayor es 60 años.

En cuanto a la perspectiva económica toma en cuenta la edad en la que debe retirarse de trabajar, al igual que en los cambios biológicos, en este aspecto existen variaciones, a nivel internacional la media es de 65 años, edad considerada también en México. Cabe mencionar 
que esta característica sólo aplica a aquellos que sean económicamente activos. Empiezan las paradojas, una persona puede presentar un alto grado de deterioro biológico y no puede jubilarse porque no ha cumplido la edad requerida, se es anciano y se deberá seguir trabajando.

\subsection{Modelo de estudio.}

Se utiliza el de Wilson (2000), ya que integra todos los fenómenos de interés: necesidades de información, comportamiento en la búsqueda y procesamiento y uso de información (utilización). Este modelo es el resultado de casi dos décadas de investigación, en 1981 Wilson postula las bases para generar una teoría sobre el comportamiento en la búsqueda, nota que el uso de la información es una de las áreas más descuidadas, sin embargo, no abunda sobre esto en toda su obra. Para 1999 publica la primera versión de su modelo de comportamiento en la búsqueda de información, en el que incluye un estadio denominado procesamiento y uso de la información, como una parte necesaria para conformar el ciclo de su modelo (WILSON, 1999).

En el año 2000 lo rehace, le da una connotación holística y lo denomina Modelo de Comportamiento Informativo (ver Fig. 2) al cual define como:

[...] la totalidad del comportamiento humano en relación con los recursos y canales de información, incluyendo la búsqueda de información activa y pasiva, y el uso de información. Integra la comunicación cara-a-cara con otros, la recepción pasiva de información como mirar anuncios de TV, sin alguna intención de actuar sobre la información dada. (WILSON, 2000, p. 49).

El punto de inicio y conclusión del modelo integra un elemento de gran relevancia, el contexto personal que determina el contexto de las necesidades de información, un estudio de usuarios debe considerar siempre el conjunto de situaciones pasadas y presentes en las que se mueven cotidianamente las personas ya que determina el tipo de problemas que tienen, las acciones que realizan para resolverlos y los procesos de uso. Incorpora una serie de variables que inciden directamente en la comunidad de adultos mayores y se alinean con el concepto de persona adulta mayor establecido: psicológicas, demográficas, papeles o roles sociales y del medio ambiente, en esta última se consideran las instituciones que los apoyan y los espacios en los que conviven.

Fig. 2 Modelo de Comportamiento Informativo. 


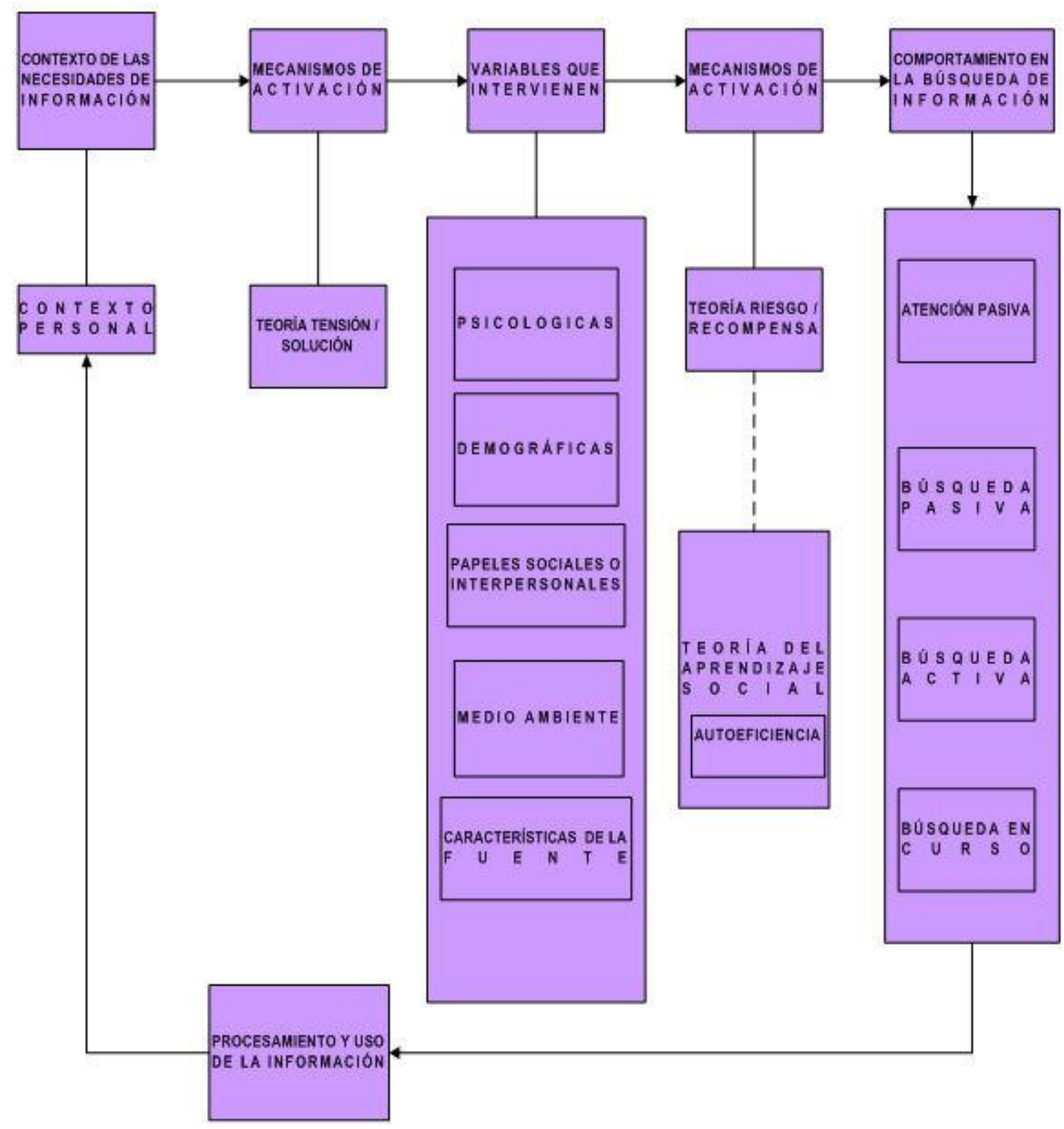

Fuente: Wilson, T. D. Human information behaviour. Informing Science Research, v. 3, n. 2, p. 53, 2000.

Establece y define las áreas en las que se basa el comportamiento informativo, importa la última procesamiento y uso de la información, la cual:

[...] consiste en los actos físicos y mentales involucrados en incorporar la información encontrada en la base de conocimiento existente en la persona. Puede incluir, actos físicos como marcar secciones de un texto para resaltar su importancia o significancia, tanto como actos mentales, por ejemplo, comparar la nueva información con el conocimiento existente (WILSON, 2000, p. 50).

A pesar de definir el uso y valorarlo como área básica de su modelo, cuando lo explica determina que es un estadio subjetivo, no directamente observable ya que está en la mente del individuo. 


\subsection{Uso de la información}

A partir de finales de los noventa del siglo pasado se han publicado resultados de investigaciones relacionadas con la utilización de la información. Existen al menos tres formas de nombrar a este fenómeno: uso del conocimiento; utilización de la información o utilización del conocimiento; esto muestra que uso y utilización e información y conocimiento son tratados como sinónimos.

Si entendemos al conocimiento como una acción y producto de procesos de la mente, se convierte en un ente abstracto y subjetivo imposible de asir o manipular, lo que hace insostenibles las frases uso o utilización del conocimiento, quedando así sólo dos maneras de mencionarlo como uso o utilización de la información. Ross J. Todd fue el primero en abordar el tema de manera sistematizada, en su artículo Back to our beginnings: information utilization, Bertram Brookes and the fundamental equation of information science (1999a) establece la relación entre el enfoque cognitivo representado por la ecuación de Brookes y el estudio de los procesos que se realizan en la mente de los individuos al utilizar la información, generalmente referida a los recursos primarios.

Establece que la forma de denominarlo es utilización debido a las diferencias semánticas entre "use" and "utilization", en español uso y utilización son sinónimos. Deriva su concepto de utilización de la información tras establecer estas premisas: alguien que hace algo con la información de manera continua; la transformación que promueve la información en los individuos, es decir, lo que les permite resolver situaciones problemáticas; y procesos que permiten a los sujetos comprometerse activamente al realizar algo con la información, toda vez que ha sido recuperada. Para él la utilización de información es un:

[...] proceso holístico que involucra procedimientos cognitivos $y$ transformaciones que ocurren dentro de la mente de los individuos cuando trabajan con la información." (1999a, p. 856)

Se aprecia que el uso es un estadio posterior a la búsqueda de la información en herramientas secundarias (generalmente) e implica la lectura y entendimiento de lo recuperado, es decir, los recursos primarios, por parte de un individuo, es menester que se haga algo con ella, la información "utilizada" debe apoyar en la solución de un problema o en la toma de alguna decisión.

Establece cinco transformaciones cognitivas que se operan en los sujetos al ser expuestos a información nueva:

- Obtener una imagen completa. Implica que se amplía el conocimiento que se tiene sobre un objeto o fenómeno para perfeccionarlo y concluirlo. 
- Obtener una imagen cambiada. La información varía los conceptos que se poseían y se logra un conocimiento transformado.

- Obtener una imagen más clara. Se desvanecen ambigüedades y dudas sobre un fenómeno.

- Obtener una imagen verificada. Al asimilar nueva información se comprueban las ideas que ya se tenían.

- Obtener una postura ante la imagen. Toda vez que se tiene una idea completa, es posible generar opiniones y defenderlas mediante la argumentación pertinente. (TODD, 1999b, pp. 15-20).

\section{Método, técnica e instrumentos}

En términos generales la investigación sigue una tendencia metodológica cualitativa. Para cubrir cada uno de los objetivos particulares se empleará un método, una técnica y un instrumento específicos:

1. Establecer el perfil de necesidades de información. Como se apuntó en los antecedentes, se han realizado estudios sobre necesidades de información en adultos mayores desde la década de los setenta del siglo pasado, los cuales han derivado en una buena cantidad de textos que hablan sobre el tema. Se está siguiendo la técnica de análisis bibliográfico ${ }^{1}$ y los instrumentos son los textos, se están analizando para eventualmente establecer las necesidades de información.

2. Identificar el comportamiento en la búsqueda y recuperación de información. Se seguirán dos caminos, el método de observación directa, cuyo instrumento será una guía de observación; y la técnica grupo focal, mediante una entrevista grupal (como instrumento).

3. Precisar los procesos cognitivos que realizan para asimilar y aplicar la información. El método será una entrevista a profundidad.

Se aprecia que esta investigación es de corte holístico, cada estadio se examina como una realidad que lleva a determinar el comportamiento informativo, esto permitirá resolver sus problemas cotidianos y minimizar su estado de vulnerabilidad.

\section{Conclusiones}

Los adultos mayores son personas cuyas características biológicas óptimas han disminuido, por esta disminución en sus funciones es posible que sufran cambios psicológicos; tanto las condiciones biológicas como psicológicas los hacen proclives a ser considerados vulnerables y afectar su aceptación social. Otro cambio significativo es dejar de ser

\footnotetext{
${ }^{1}$ De aquí la frase "En términos generales", esta técnica no es de corte cualitativo.
} 
económicamente activos, sus ingresos disminuyen y es posible que dependan de otros para su manutención.

Todas estas condiciones pueden ser paliadas mediante el uso de la información que necesitan, a nivel internacional se han hecho investigaciones que han abordado ciertos aspectos de esta comunidad en relación con la información, sin embargo, no se ha analizado el fenómeno de manera integral, en México no existen estudios. Esta investigación aborda su comportamiento informativo, entendido desde el modelo de Wilson como la totalidad de las actividades y elementos que vinculan a la información con cualquier comunidad, en este caso personas adultas mayores. Contexto, variables demográficas, psicológicas, sociales, roles sociales, medio ambiente, espacios, legislación, instituciones y programas institucionales son los elementos fundacionales para desarrollarla.

El uso es concebido como la transformación cognitiva que se genera en los sujetos al leer, analizar, sintetizar, asimilar y acomodar la nueva información en su acervo de conocimientos. Se puede afirmar que, comprendido de esta manera, el fenómeno ha sido poco examinado, requiere seguir un enfoque cognitivo y una metodología cualitativa.

Con esta investigación se espera:

Entender el comportamiento de los adultos mayores ante la información.

Resolver problemas cotidianos: salud, convivencia, económicos, de participación, trámites y movimiento.

- Sugerir alternativas de servicios que aumenten el bienestar de la vida de esta población, la cual es poco atendida y en ocasiones marginada por las instituciones de información.

Incidir en la generación de políticas públicas.

Cubrir la sugerencia de la ONU sobre la generación de investigación sobre estas comunidades.

Y finalmente que haya presencia de los especialistas de la información ante las instancias que generan planes y programas para lograr su bienestar.

\section{Bibliografía}

ALLEN, B.; WILKINSON, M. A. What do 'senior citizens' want from public libraries? Canadian Library Journal, v. 47, n. 2, p. 105-110, 1990.

BARNETT, M. J. Factors influencing older adults' patterns of information acquisition. Denton, Texas: The author. 1989. 
BARRETT, J. The information needs of elderly, disabled elderly people, and their careers. United Kingdom: Disability Information Trust, 2000.

BARRETT, J. Support and information needs of older and disabled older people in the UK Original Research Article. Applied Ergonomics, v. 36, n. 2, p. 177-183, 2005. Disponible en: https://ac.elscdn.com/S0003687004001371/1-s2.0-S0003687004001371- main.pdf? tid=8707d712-18d511e8-be5e-00000aab0f27\&acdnat=1519416781 d80225f785c5d5f4b2459a9b6c1f9998

BELKIN, N. J. Information concepts for information science. Journal of Documentation, v. 34, n. 1, p. 55-85, 1978.

BELKIN, N. J. The cognitive viewpoint in information science. Journal of Information Science, v. 6, p. 11-15, 1990.

BELKIN, N. J.; ODDY, R. N.; BROOKS, H. M. ASK for information retrieval. Part I: Background and theory; Part II: Results of a design study. Journal of Information Science, v.38, n. 2-3, p. 61-71; p. 145-164, 1982.

BROOKES, B. C. Robert Fairthorne and the scope of information science. Journal of Documentation, v. 30, n. 2, p. 139-152, 1974.

BROOKES, B. C. The fundamental problem of information science. Informatics, v. 2, p. 42-49, 1975.

BROOKES, B. C. The developing cognitive view point in information science. In: MEY, M. de (ed.). International Workshop on the Cognitive Viewpoint. Gent: University of Ghent, p. 195-203, 1977.

BROOKES, B. C. The foundations of information science. Part I. Philosophical aspects: classes of things and the challenge of human individuality. Journal of Information Science, v. 2, p. 125-133, 1980.

BROOKES, B. C. The foundations of information science. Part IV: Information Science the changing paradigm. Journal of Information Science, v. 3, p. 3-12, 1981.

BROWNSELL, S.; BLACKBURN, S.; HAWLEY, M. User requirements for an ICT-based system to provide care, support and information access for older people in the community. Journal of Assistive Technologies, v. 6, n. 1, 2012. Disponible en: http://www.emeraldinsight.com/doi/pdfplus/10.1108/17549451211214328

$\mathrm{CHU}$, A. et al. Partnering with Seniors for Better Health: Computer Use and Internet Health Information Retrieval among Older Adults in a Low Socioeconomic Community. Journal of the Medical Library Association, v. 97, n. 1, p. 12-20, 2009.

CONTACTO HOY EDICIÓN MUNDIAL. Disponible en: https://contactohoy.com.mx/mas-de-12millones-de-personas-en-mexico-son-adultos-mayores-inegi/

DEE, M.; BOWEN, J. Library service to older people. London: British Library, 1986.

GODFREY, M.; JOHNSON, O. Digital circles of support: Meeting the information needs of older people. Computers in Human Behavior, v. 25, n. 3, p. 633-642, 2009. Disponible en: https://ac.els-cdn.com/S0747563208001702/1-s2.0-S0747563208001702-main.pdf? tid=spdf84995bbb-e822-4b86-9b13e6bc60cb4021\&acdnat $=1519416628$ fdf0608e402e0eb7b02925f43e291c25

GOODMAN, J.; BREWSTER, S.; GRAY, P. Memory aids for older people. In: BRITISH HCI CONFERENCE, $16^{\text {th }}$, London, UK. Proceedings. London: British HCl Group, 2002. Disponible en: http://citeseerx.ist.psu.edu/viewdoc/download?doi=10.1.1.135.952\&rep=rep1\&type=pdf

HEIM, K. M.; WALLACE, D. P. Adult services: An enduring focus for public libraries. Chicago: American Library Association, 1990. 
HERNÁNDEZ SALAZAR, P. Transformación de la metodología para estudiar a los usuarios de la información. En: RíOS ORTEGA, J. \& RAMÍREZ VELÁZQUEZ, C. A. (eds.). Naturaleza y método de la investigación bibliotecológica y de la información. México: UNAM, Instituto de Investigaciones Bibliotecológicas y de la Información, 2014. p. 209-252. [ebook] Disponible en:

http://iibi.unam.mx/archivistica/naturaleza metodo inv bibl corto.pdf

IHM, J.; HSIEH, Y. P. The implications of information and communication technology use for the social well-being of older adults. Information, Communication \& Society, v. 18, n. 10, p. 11231138, 2015.

INDEX MUNDI. [online] Disponible en: https://www.indexmundi.com/map/?v=30

MEXICO. DISTRITO FEDERAL. ASAMBLEA LEGISLATIVA. Ley de albergues privados para personas adultas mayores del Distrito Federal. (2009). [pdf] Disponible en: http://aldf.gob.mx/archivoc3c84deb4108970eaee0851d7bed9198.pdf

MEXICO. PRESIDENCIA DE LA REPUBLICA. Ley de los Derechos de las Personas Adultas Mayores. (2016). [pdf] Disponible en: https://www.gob.mx/cms/uploads/attachment/file/175189/245 221116.pdf

MEXICO. SECRETARÍA DE DESARROLLO SOCIAL. Guía de programas sociales. [online] Disponible en: https://www.gob.mx/cms/uploads/attachment/file/119689/GPS 2016.pdf

NIEMELA, R.; HUOTARI, M. L.; KORTELAINEN, T. Enactment and use of information and the media among older adults. Library \& Information Science Research, v. 34, n. 3, p. 212-219, 2012.

ORGANIZACIÓN DE LAS NACIONES UNIDAS [ONU]. Plan de Acción Internacional de Viena sobre el Envejecimiento. $1982 . \quad$ [online] Disponible en: http://www.sld.cu/galerias/pdf/sitios/gericuba/plan de accion internacional de viena sobr e el envejecimiento.pdf

ORGANIZACIÓN DE LAS NACIONES UNIDAS [ONU]. Principios de las Naciones Unidas en favor de las Personas de Edad. 1991. [online] Disponible en: https://www.acnur.org/5b6caf814.pdf

ORGANIZACIÓN DE LAS NACIONES UNIDAS [ONU]. Proclamación sobre el Envejecimiento. 1992. [online] Disponible en: https://undocs.org/es/A/RES/47/5

ORGANIZACIÓN MUNDIAL DE LA SALUD [OMS]. 10 datos sobre el envejecimiento y la saludo. Disponible en: https://www.who.int/features/factfiles/ageing/es/

SCHELLING, H. R.; SEIFERT, A. L'utilisation de l'internet par les personnes âgées. 2010. Disponible en: https://www.zfg.uzh.ch/dam/icr:00000000-44fb-2214-000000000d622506/IKT Kurzbericht 20103 f bf.pdf

SMITH, A. Older Adults and Technology Use. 2014. Disponible en: http://assets.pewresearch.org/wp-content/uploads/sites/14/2014/04/PIP Seniors-and-TechUse 040314.pdf

TAK, S. H.; HONG, S.H. Use of the Internet for health information by older adults with Arthritis. Orthopaedic Nursing, v. 24, n. 2, p. 134-138, 2005.

TODD, R. J. Information Utilization: A Cognitive Analysis of how Girls Utilize Drug Information Based on Brookes' Fundamental Equation $[\mathrm{K}[\mathrm{S}]+\Delta \mathrm{I}=\mathrm{K}[\mathrm{S}+\Delta \mathrm{S}]$. In: Information seeking in context. London: Taylor Graham. 1996.

TODD, R. J. Back to our beginnings: information utilization, Bertram Brookes and the fundamental equation of information science. Information Processing and Management, v. 35, p. 851-870, 1999a. 
TODD, R. J. Utilization of Heroin Information by Adolescent Girls in Australia: A Cognitive Analysis. Journal of the American Society for Information Science, v. 50, n. 1, p. 10-23, 1999b.

WICKS, D. A. Older Adults and Their Information Seeking. Behavioral \& Social Sciences Librarian, v. 22, n. 2, p. 1-26, 2004 . [online] Disponible en: https://www.tandfonline.com/doi/abs/10.1300/J103v22n02 01

WILSON, T. D. On user studies and information needs. 1981. Journal of Documentation, v. 37, n. 1, p. 3-15. [online] Disponible https://pdfs.semanticscholar.org/c402/314407034f3670ce1db0a41d07cee0349a1e.pdf

WILSON, T. D. Models in information behaviour research. 1999. Journal of Documentation, v. 55, n. 3, p. 249-270. [online] Disponible en: https://www.researchgate.net/publication/228784950 Models in Information Behaviour Re search

WILSON, T. D. Human information behavior. Information Science, [online] v. 3, n. 2, p. 49-55, 2000. Disponible en: http://inform.nu/Articles/Vol3/v3n2p49-56.pdf

WILSON, T. D.; WALSH, C. Information behavior: an interdisciplinary perspective. Information Processing \& Management, v. 33, n. 4, p. 551-572, 1997. 